\title{
SARS-CoV-2 and Advanced HIV Infection
}

\section{Dear Editor,}

There have been conflicting reports on whether human immunodeficiency virus (HIV) infection is associated with increased mortality or risk of severe disease in COVID-19 infections. While most studies suggest that this is not the case, there are new evidence to suggest that co-infection could be associated with increased mortality. ${ }^{1-6}$ We describe a patient presenting with newly diagnosed AIDS, multiple opportunistic infections and COVID-19 infection.

A 48-year-old transgender woman was admitted for cough, shortness of breath and diarrhoea of 3 weeks' duration. On examination, she was afebrile, tachycardic, and had a blood pressure of $77 / 56 \mathrm{mmHg}$ and oxygen saturation of $69 \%$ on room air. She was cachectic with oral thrush. Crepitations were heard over the right lung base.

She was intubated and admitted to the intensive care unit (ICU). Computed tomography of her chest showed multiple patchy ground-glass opacities bilaterally. Her HIV screen was positive, with a viral load of 28,700 copies $/ \mathrm{ml}$ and $\mathrm{CD} 4$ count of $<20$ cells $/ \mu 1$. Endotracheal samples for both SARS-CoV-2 polymerase chain reaction (PCR) and Pneumocystis jirovecii PCR were positive. The initial cycle threshold value of her SARS-CoV-2 PCR on admission was 25.45. Blood culture was positive for Salmonella enteritidis. She was initiated on trimethoprim-sulfamethoxazole and prednisolone for severe pneumocystis pneumonia (PCP) and ceftriaxone for Salmonella enteriditis bacteraemia. Our patient was considered for recruitment into an ongoing remdesivir treatment trial, but was not eligible in view of her impaired renal function. There was rapid clinical improvement with antimicrobial therapy and our patient was extubated after 4 days. Antiretroviral therapy (ART) was initiated after 1 week of admission. After 8 weeks of inpatient physiotherapy, the patient was discharged.

The current available data shows conflicting evidence on whether COVID-19 and HIV co-infection are associated with greater mortality or severe disease. A case series in Spain reported a mortality rate of $4 \%$ among 51 HIV-infected individuals with COVID-19, which was lower than that of the general population, although $25 \%$ had severe disease and $12 \%$ required ICU admission. ${ }^{6}$ No patients were reported to have other opportunistic infections. However, a prospective observational study in the UK showed that mortality was higher in HIV-infected individuals with COVID-19 infection, with a reported hazard ratio of $1.63 .{ }^{7}$ In addition, a case control study in the UK suggested that there is a three-fold higher risk of COVID-19 death in people living with a HIV infection, compared to those without. ${ }^{2}$

Despite multiple concomitant infections in an immunocompromised state, our patient improved rapidly. One possible reason could be the use of corticosteroids. In the Randomised Evaluation for COVID-19 Therapy (RECOVERY) trial, dexamethasone conferred mortality benefit in patients with COVID-19 infection requiring oxygenation. ${ }^{7}$ Corticosteroids are given in conjunction with PCP therapy to reduce incidence of mortality and respiratory failure associated with severe PCP. ${ }^{8}$ While our patient was not given dexamethasone, she was on high-dose prednisolone as part of her treatment for severe PCP. This could possibly have an effect of mitigating the severity of her COVID-19 infection.

Another possibility for her clinically milder course of COVID-19 infection could be related to her low CD4 cell counts. In a case report by $\mathrm{Xu}$ et al. of a patient with severe COVID-19 infection and acute respiratory distress syndrome (ARDS), they found that while there were low levels of CD4 and CD8 T cells in the peripheral circulation, these lymphocyte subsets were hyperactivated. ${ }^{9}$ There was also an increased concentration of highly inflammatory CCR6+ TH17 in CD4 $\mathrm{T}$ cells, suggesting that $\mathrm{T}$ cell overactivation could have contributed to the severe immune injury in the patient. ${ }^{9}$ Others have also demonstrated that severe COVID-19 disease was associated with a greater degree of pathological CD4 and CD8 T cell activation. ${ }^{10}$ In our patient, the CD4 cell count of $<20$ cells/ $\mu$ l may have dampened the inflammatory response to COVID-19 infection, thereby reducing immune injury and resulting in relatively rapid clinical improvement. 
There are potential concerns for immune reconstitution inflammatory syndrome to COVID-19 upon initiation of ART, which may result in ARDS. The ideal timing for initiation of ART in these cases is unknown. We chose to initiate ART once the patient no longer required supplemental oxygen. No adverse effect from the initiation of ART has been observed during the 8 weeks of hospitalisation.

In conclusion, more data is required to understand the impact of AIDS and its associated therapies on COVID-19 infection. It is important to consider ruling out COVID-19 infection in patients with HIV presenting with opportunistic infections, especially PCP, as it can be difficult to differentiate between the 2 infections or rule out co-infections.

\section{REFERENCES}

1. Geretti AM, Stockdale AJ, Kelly SH, et al. Outcomes of COVID-19 related hospitalization among people with HIV in the ISARIC WHO Clinical Characterization Protocol (UK): a prospective observational study. Clin Infect Dis 2020. doi: 10.1093/ cid/ciaa1605.

2. Bhaskaran K, Rentsch CT, MacKenna B, et al. HIV infection and COVID-19 death: a population-based cohort analysis of UK primary care data and linked national death registrations within the OpenSAFELY platform. The Lancet HIV 2021;8:e24-32.

3. Altuntas Aydin O, Kumbasar Karaosmanoglu H, Kart Yasar K. HIV/ SARS-CoV-2 co-infected patients in Istanbul, Turkey. J Med Virol 2020;92:2288-90
4. Zhu F, Cao Y, Xu S, et al. Co-infection of SARS-CoV-2 and HIV in a patient in Wuhan city, China. J Med Virol 2020;92:529-30.

5. Blanco JL, Ambrosioni J, Garcia F, et al. COVID-19 in patients with HIV: clinical case series. Lancet HIV 2020;7:e314-6.

6. Vizcarra P, Pérez-Elías MJ, Quereda C, et al. Description of COVID-19 in HIV-infected individuals: a single-centre, prospective cohort. Lancet HIV 2020;7:e554-64.

7. Horby P, Lim WS, Emberson JR, et al. Dexamethasone in hospitalized patients with Covid-19 - Preliminary report. N Engl J Med 2020. doi: 10.1056/NEJMoa2021436.

8. Ewald H, Raatz H, Boscacci R, et al. Adjunctive corticosteroids for Pneumocystis jiroveci pneumonia in patients with HIV infection. Cochrane Database Syst Rev. 2015;2015:Cd006150.

9. Xu Z, Shi L, Wang Y, et al. Pathological findings of COVID-19 associated with acute respiratory distress syndrome. Lancet Respir Med 2020;8:420-2.

10. Qin C, Zhou L, Hu Z, et al. Dysregulation of immune response in patients with coronavirus 2019 (COVID-19) in Wuhan, China. Clin Infect Dis 2020;71:762-8.

Chiaw Yee Choy, ${ }^{1,2}$ MBBS (Spore), MRCP (UK), Chen Seong Wong, ${ }^{1,2,3}$ MBBS (S'pore), MRCP (UK)

${ }^{1}$ National Centre for Infectious Diseases, Singapore

${ }^{2}$ Department of Infectious Diseases, Tan Tock Seng Hospital, Singapore

${ }^{3}$ Yong Loo Lin School of Medicine, National University of Singapore, Singapore

Address for Correspondence: Dr Chiaw Yee Choy, National Centre for Infectious Diseases; Department of Infectious Diseases, Tan Tock Seng Hospital, 16 Jln Tan Tock Seng, Singapore 308442.

Email: chiawyee_choy@ncid.sg 